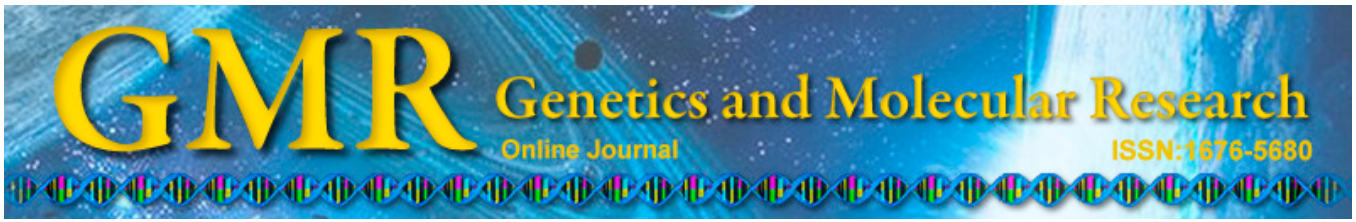

\title{
Correlation of E6 and E7 levels in high-risk HPV16 type cervical lesions with CCL20 and Langerhans cells
}

\author{
B. Jiang and M. Xue \\ Department of Gynecology and Obstetrics, the Third Xiangya Hospital, \\ Central South University, Changsha, China \\ Corresponding author: M. Xue \\ E-mail: MinXueghj@163.com
}

Genet. Mol. Res. 14 (3): 10473-10481 (2015)

Received January 25, 2015

Accepted May 8, 2015

Published September 8, 2015

DOI http://dx.doi.org/10.4238/2015.September.8.8

\begin{abstract}
The human papillomavirus(HPV)16E6 andE7 correlation with chemokine ligand (CCL)20 expression and Langerhans cells (LCs) in cervical lesions was investigated. We enrolled 43 patients with surgically treated cervical lesions from the Department of Gynecology in our hospital, and 20 controls without cervical lesions. Subjects were divided by pathology: HPV16(-) and HPV16(+) normal cervical groups $(\mathrm{N}=10$ each), and HPV16(+) cervical intraepithelial neoplasia (CIN), cervical invasive carcinoma $(\mathrm{N}=15$ each), and in situ carcinoma ( $\mathrm{N}$ $=13$ ) groups. E6, E7, the LC surface marker CD1a, and CCL20 were analyzed by immunohistochemistry. E6 and E7 in HPV16-type lesions were correlated with CCL20 and LCs. The average high power field cell numbers of $\mathrm{CD} 1 \mathrm{a}^{+} \mathrm{LCs}$ in the HPV(-) and $\mathrm{HPV}(+)$ normal cervix groups, and the CINI-II, CINIII in situ and cervical carcinoma groups were $22.89 \pm 4.84,13.7 \pm 2.26,9.2 \pm 1.68,5.9 \pm 1.59$, and $5.5 \pm 1.58$, respectively. Significant between-group differences existed except between cervical carcinoma and CINIII groups $(\mathrm{P}<0.05)$. CCL20 $0^{+}$ rates in each group were $70,60,60,15.38$, and $13.33 \%$, respectively.
\end{abstract}


E6/E7-positive expression rates in each group were 20/20, 66.7/66.7, $76.9 / 69.2$, and $86.67 / 73.3 \%$, respectively. CCL20 was positively correlated with CD1a $(\mathrm{r}=0.649)$, and negatively correlated with E7 $(\mathrm{r}=$ $-0.946)$ and E6 $(r=-0.949)$. CD1a was negatively correlated with E6 ( $r$ $=-0.632)$ and E7 $(r=-0.632)$. Downregulation of CCL20 leading to LC decline is a key factor in cervical lesions. High-risk HPV-type lesions might inhibit the chemokine CCL20 through E6 and E7 to escape the immune response.

Key words: Cervix; CCL20; Langerhans cells; E6; E7

\section{INTRODUCTION}

Cervical cancer is a common malignant tumor and has the highest incidence among gynecological tumors. The rate of cervical cancer in women is rising each year, and it has become a large threat to women's health and a substantial burden on the global economy (Hussain et al., 2014; Skaaby et al., 2014). Its morbidity and mortality have remained at high levels in China (Du et al., 2014; Xu et al., 2014). Although the occurrence and development of cervical cancer is a long-term and gradual process caused by multiple carcinogenic factors, persistent high-risk human papillomavirus (HPV)-type infection represents one of the clearest etiologies. Persistent expression of the E6 and E7 proteins of the high-risk type HPV16 virus is a key risk factor for cervical cell transformation and the maintenance of a malignant phenotype (Gan et al., 2014; He et al., 2014), and represents the primary mechanism by which the virus escapes host cell immune surveillance after HPV infection. Thus, the immune escape mechanism of HPV has become the focus of current research (Niebler et al., 2013; Nahvijou et al., 2014).

Langerhans cells (LCs) are primarily expressed in the epidermis and are a kind of immature dendritic cell. LCs are the main immune cell to act as antigen-presenting cells for presentation of the HPV virus to trigger an immune response (Rios-Yuil et al., 2014).

LC migration depends on the chemokines in the environment and the specific chemokine receptors on the LCs. Chemokine ligand (CCL)20 is a chemokine that is formed and secreted by keratinocytes, and LCs can express the CCL20 receptor chemokine receptor (CCR)6 (Amador-Molina et al., 2013; Da Silva et al., 2014). LCs only express CXCR4 and CCR6 in normal skin; normal skin keratin formation cells and venous endothelial cells express CCL20; while myeloid dendritic cell precursor cells lose their ability to react to the CCR2 ligand but gain the ability to react to the CCR6 ligand, CCL20, when they differentiate into LCs. It was thought that CCL20 might play an important role in regulating LCs and the selective entry of their precursor cells into the epidermis (Nakayama et al., 2011; Sperling et al., 2012). There is still a lack of information regarding whether CCL20 can influence HPV infection. It has been suggested that HPV infection can downregulate CCL20 (Wang et al., 2010); however, whether the HPV16 E6 and E7 proteins are correlated with LCs and CCL20 is still controversial. In this study, we tried to investigate the correlation of E6 and E7 in high-risk HPV16-type infection with CCL20 and LCs within cervical lesions. 


\section{MATERIAL AND METHODS}

\section{Subjects}

We enrolled 43 patients with cervical lesions who had been treated by surgery in the Department of Gynecology in the Third Xiangya Hospital of Central South University; the average patient age was $46.38 \pm 6.61$ years. There were 15 patients in the cervical intraepithelial neoplasia (CIN)I-CINII group, 13 in the CINIII-carcinoma in situ group, and 15 patients in the cervical invasive carcinoma group. All of the patients were infected by HPV16 alone and the surgery represented their initial treatment. All specimens were diagnosed by pathology and patients had received no preoperative chemotherapy or radiation therapy intervention. Exclusion criteria included metastatic tumor, cervical tumor combined with other tumors, a history of preoperative radiotherapy or chemotherapy, combined autoimmune disease, and a history in receipt of oral immune inhibitors. An additional 20 subjects with normal cervixes were collected with the average age of $37.8 \pm 3.56$ years. Among these, 10 were in the HPV(-) normal cervix group and 10 were in the HPV16(+) normal cervix group. The general clinical information showed no statistical difference between the two groups $(P>0.05)$. Subjects were informed and had signed informed consent, and the research content was approved by the hospital Ethics Committee.

\section{Main instruments and reagents}

The CD1a monoclonal antibody for immunohistochemistry was bought from Maxim Biotechnology Co., Ltd. (Fuzhou, China). The CCL20 monoclonal antibody, E6 protein monoclonal antibody, and E7 protein monoclonal antibody were obtained from Bioss Biological Company (Beijing, China). The streptavidin-peroxidase (SP) kit was bought from the Zhongshan Golden Bridge Bio-Technology Co., Ltd. (Beijing, China).

\section{Methods}

\section{Grouping}

According to their cervical lesion progression and HPV16 infection status, the subjects were divided into four groups: HPV(-) normal cervical group $(\mathrm{N}=10)$, HPV16(+) cervical normal group $(\mathrm{N}=10)$, HPV16(+) CIN group $(\mathrm{N}=28)$, and HPV16 $(+)$ cervical invasive carcinoma group $(\mathrm{N}=15)$. No significant difference of the general information was detected among each group $(\mathrm{P}>0.05)$.

\section{Immunohistochemistry}

The immunohistochemical SP method was utilized to detect the expression of LCs, and E6, E7, and CCL20 proteins. Tissue samples were collected from the surgical resection, fixed in $4 \%$ paraformaldehyde, embedded in paraffin, and sectioned $(5 \mu \mathrm{m})$. Paraffin sections were dewaxed and the endogenous peroxidase was blocked with $3 \% \mathrm{H}_{2} \mathrm{O}_{2}$ solution. After being washed by $0.01 \mathrm{M}$ sodium citrate buffer, $\mathrm{pH}$ 6.0, the antigen was repaired. Monoclonal antibodies for E6, E7, CD1a, or CCL20 (Santa Cruz, USA) were applied at 1:500 dilutions for 
incubation overnight at $4^{\circ} \mathrm{C}$. The slices were incubated with goat anti-mouse IgG conjugated with horseradish peroxidase (Invitrogen, USA) and colorized by DAB.

The results were evaluated as follows: CD1a-positive yellow or brown cells with irregular dendritic protrusions, identified as Langerhans cells, were counted at high magnification under a microscope. The immunoreactive score was applied to evaluate protein and CCL20 expression. Five fields of vision were selected on each slide for evaluation based on brown positive intensity and area. For each field of vision, positive intensity with no coloring, light yellow, weak brown, and brown were scored $0,1,2$, and 3 points, respectively; positive staining area with no color, color area less than $25,25-50 \%, 50-75 \%$, and $>75 \%$ were scored $0,1,2$, and 3 points, respectively. The two scores were added and the five view score results were averaged. A score of 0-1 represented a negative (-) result, 2 represented weakly positive $(+), 3-4$ represented moderately positive $(++)$, and 5-6 represented strong positive $(+++)$; scores of 2-6 represented positive results.

\section{Statistical analysis}

Enumeration data was analyzed by the chi-square test and measurement data are reported as means \pm standard deviation. ANOVA and $t$-test were chosen for analysis when appropriate. Logistic regression was applied for correlation analysis between E6 and E7, CCL20, and CD1a protein results. $\mathrm{P}$ values of less than 0.05 were considered to be significant.

\section{RESULTS}

\section{CD1a expression}

$\mathrm{CD}_{1} \mathrm{a}^{+} \mathrm{LCs}$ were primarily distributed in the membranes or cytoplasm of the middle and basic layers in the squamous epithelium, with irregular dendritic protrusions on morphology. The average hight power fields of $\mathrm{CD} 1 \mathrm{a}^{+} \mathrm{LCs}$ in the HPV(-) normal cervix group, the $\mathrm{HPV}(+)$ normal cervix group, and the CINI-II, CINIII in situ carcinoma, and cervical carcinoma groups were $22.89 \pm 4.84,13.7 \pm 2.26,9.2 \pm 1.68,5.9 \pm 1.59$, and $5.5 \pm 1.58$, respectively (Figure 1). The difference between each group was statistically significant except between the cervical carcinoma and CINIII groups $(\mathrm{P}<0.05)$.

\section{CCL20 expression}

CCL20 was primarily expressed in the cytoplasm or membrane of the squamous epithelium in normal cervix. It was expressed less in the CINIII-carcinoma in situ and cervical invasive carcinoma tissues (Figure 2). The positive rate of CCL20 in each group was 70, 60, $60,15.38$, and $13.33 \%$, respectively. Significant differences were observed between the normal cervix and cervical cancer groups, the normal cervix and CINIII-carcinoma in situ groups, the CINI-CINII and cervical cancer groups, and the CINI-CINII and CINIII-carcinoma in situ groups $(\mathrm{P}<0.05)$.

\section{E6 protein expression}

E6 protein was predominantly expressed in the nucleus and only weak signals in cy- 
toplasm. It exhibited little or no expression in normal cervix, but showed strong expression in CIN and in cervical cancer (Figure 3). The positive expression rate of the E6 protein in each group was 20,66.7, 76.9, and 86.67\%, respectively. Significant differences were found between the expression in the normal cervix group and the CINI-CINII group, the CINIIIcarcinoma in situ group, and the cervical cancer group $(\mathrm{P}<0.05)$.
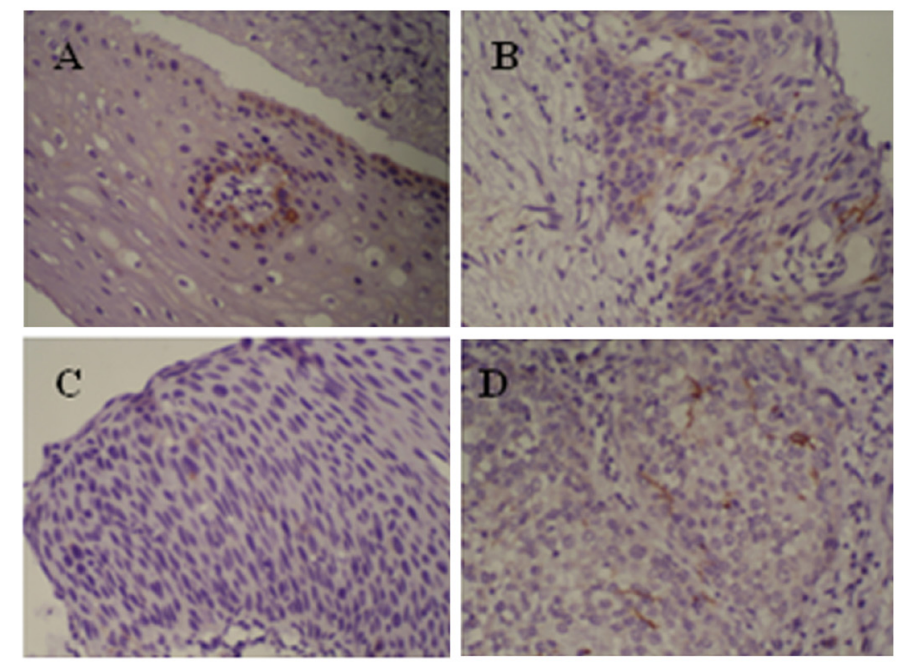

Figure 1. CDla expression in patients with cervical lesions and controls. A. CD1a expression in the normal cervix group; B. CD1a expression in the cervical intraepithelial neoplasia (CIN)I group; C. CD1a expression in the CINIII group; D. CD1a expression in the cervical carcinoma group. Immune reactivity was visualized by DAB substrate (brown) and was counter-stained with hematoxylin (blue). Magnification, 200X.
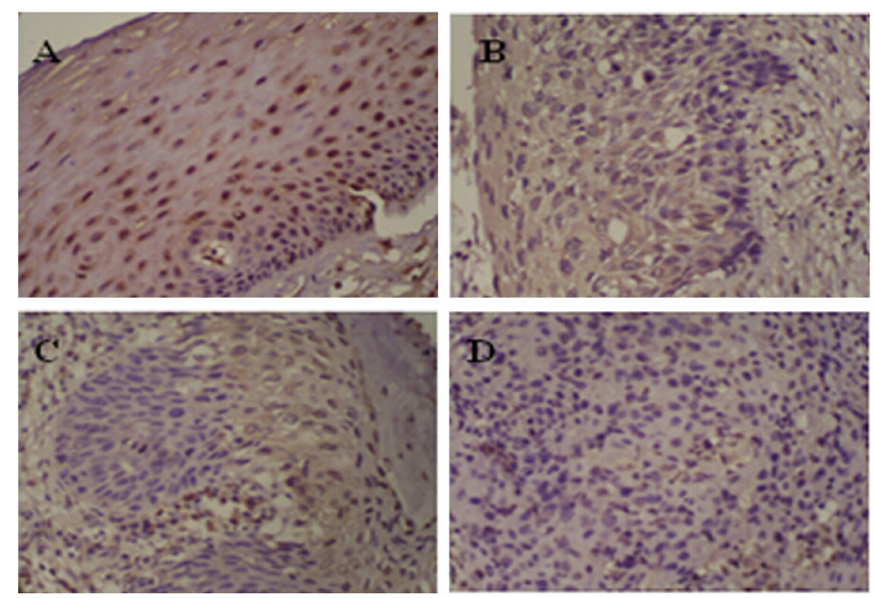

Figure 2. CCL2 expression in patients with cervical lesions and controls. A. CCL2 expression in the normal cervix group; B. CCL2 expression in the cervical intraepithelial neoplasia (CIN)I group; C. CCL2 expression in the CINIII group; D. CCL2 expression in the cervical carcinoma group. Immune reactivity was visualized by DAB substrate (brown) and was counter-stained with hematoxylin (blue). Magnification, 200X. 

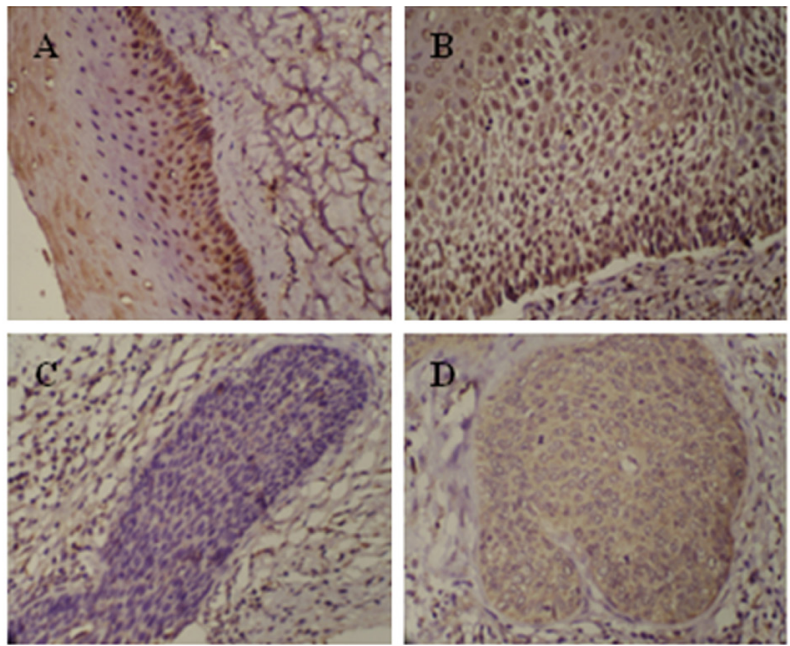

Figure 3. E6 protein expression in patients with cervical lesions and controls. A. E6 protein expression in the normal cervix group; B. E6 protein expression in the cervical intraepithelial neoplasia (CIN)I group; C. E6 protein expression in the CINIII group; D. E6 protein expression in the cervical carcinoma group. Immune reactivity was visualized by DAB substrate (brown) and was counter-stained with hematoxylin (blue). Magnification, 200X.

\section{E7 protein expression}

E7 protein expressed mainly in the nucleus but only weakly in cytoplasm. It exhibited little or no expression in normal cervical tissue, but exhibited strong expression in CIN and in cervical cancer (Figure 4). The positive expression rate of E6 protein in each group was 20, $66.7,69.2$, and $73.3 \%$, respectively. The normal cervix group showed significant differences with the other groups for E7 expression levels $(\mathrm{P}<0.05)$.
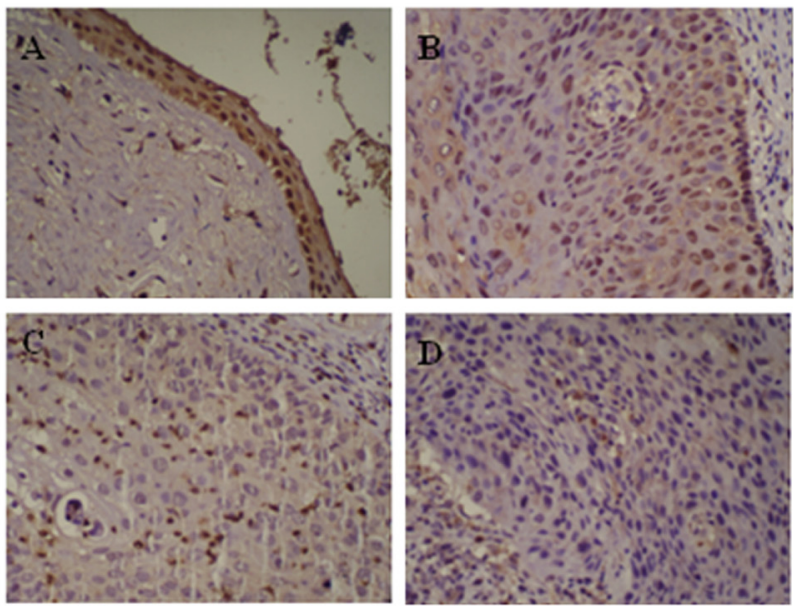

Figure 4. E7 protein expression in patients with cervical lesions and controls. A. E7 protein expression in the normal cervix group; B. E7 protein expression in the cervical intraepithelial neoplasia (CIN)I group; C. E7 protein expression in the CINIII group; D.. E7 protein expression in the cervical carcinoma group. Immune reactivity was visualized by DAB substrate (brown) and was counter-stained with hematoxylin (blue). Magnification, 200X. 


\section{Correlation of CD1a and CCL20 with E6 and E7}

The correlations of CD1a and CCL20 with E6- and E7-positive results were further analyzed. CCL20 was positively correlated with CD1a $(r=0.649)$, and significantly negatively correlated with E7 and E6 $(r=-0.946, r=-0.949, \mathrm{P}<0.05)$, respectively. The CD1apositive rate was also obviously negatively correlated with E6 and E7 $(\mathrm{r}=-0.632, \mathrm{r}=-0.632$, $\mathrm{P}<0.05)$, respectively.

\section{DISCUSSION}

Multiple factors including bacteria, viruses, microbes, precocious sexuality, promiscuity, multiple birth, and genetic susceptibility can cause cervical lesions and even cervical cancer (Zamaniah et al., 2014; Zeng et al., 2014). Numerous epidemiologic and molecular biology studies have confirmed that the HPV is closely related to the occurrence and development of genital tract epithelial malignant tumors and precancerous lesions. In particular, the persistent infection with high-risk HPV has been identified as the main etiological factor for cervical cancer. HPV-DNA can be detected in nearly $99.7 \%$ of cervical cancers. At present, the pool of individuals with HPV infection is becoming younger, and studies have shown that HPV16 exhibits the strongest pathogenic infection (Nessa et al., 2014). The persistent expression of the HPV proteins E6 and E7 is a high risk factor for cervical cancer. To date, many mechanisms of HPV carcinogenesis have been investigated; for example, the oncogenes E6 and $E 7$ can promote tumorigenesis through a P53-independent pathway, and HPV can cause cells to undergo transformation through the activation of telomerase. On the other hand, it has also been found that HPV infection can escape immune surveillance and response. Thus, research on the mechanisms underlying the latter phenomenon has important significance (Durzynska, 2014).

LCs, as important antigen-presenting cells for the capture and management of the HPV virus, exist in the epidermis. They can migrate from the epidermis to regional lymph nodes through the basement membrane of the skin and via the lymph channels, and can develop into finger dendritic cells. The number and density of LCs have been shown to be significantly reduced in the presence of HPV infection. HPV can limit the immune response by reducing LC number and density, which allows the persistent HPV infection of cervical epithelial cells. Decreased chemokine release mediated by HPV16 infection might also be an important mechanism underlying LC number reduction. The expression of cytokines such as TGF- $\beta 1$ is reduced in precancerous lesions of the uterine cervix. TGF- $\beta 1$ can promote LC progenitor cells to enter the skin and induce their differentiation. Downregulation of TGF- $\beta 1$ can affect LC infiltration and maturation in the infected area directly. HPV infection can also reduce the expression of IL- $1 \alpha$, IL-1 $\beta$, and granulocyte-macrophage colony stimulating factor (GM-CSF), factors crucial for LC migration and maturation (Ancuţa et al., 2014). GM-CSF is a cytokine that can mediate the gathering of LCs at the epidermis and is decreased significantly in cervical epithelium infected by HPV. Thus, LC number reduction after HPV infection is closely related to the decrease of chemokines. CCL20 is only expressed in inflammatory epithelial and mucosal tissue. CCR6, as the single receptor with high affinity for CCL20, is primarily expressed on the surface of LCs, and on activated T- and B-cells. In combination with its specific receptor CCR6, CCL20 plays a crucial role in the process of LC gathering and chemotaxis in cervical epithelial tissue (Giannini et al., 2002; Guess and McCance, 2005; 
Caberg et al., 2009). Our results confirmed that LC numbers were reduced in accordance with the severity of the cervical lesions; CCL20 was decreased by HPV infection; and E6 and E7 protein was also upregulated in proportion with the severity of the disease. The results demonstrated that CCL20 is therefore positively correlated with CD1a; E6 and E7 are negatively correlated with CCL20; and CD1a showed a negative correlation with E6 and E7.

In summary, our research confirmed that downregulation of CCL20 leading to LC decline is a key factor in cervical lesions. High-risk HPV-type infection might inhibit the chemokine CCL20 through E6 and E7 to escape the immune response. This finding might provide the basis for further investigation into the mechanism of HPV immune response evasion in cervical lesions.

\section{Conflict of interest}

The authors declare no conflict of interest.

\section{ACKNOWLEDGMENTS}

We thank the anonymous reviewers for reviewing this manuscript.

\section{REFERENCES}

Amador-Molina A, Hernândez-Valencia JF, Lamoyi E, Contreras-Paredes A, et al. (2013). Role of innate immunity against human papillomavirus (HPV) infections and effect of adjuvants in promoting specific immune response. Viruses 5: 2624-2642.

Ancuța E, Buțureanu S, Zugun-Eloae F, Anton CR, et al. (2014). Potential value of in situ cellular immune response in HPV subtype 16 and 18 positive cervical cancer. Rom. J. Morphol. Embryol. 55: 817-822.

Caberg JH, Hubert P, Herman L, Herfs M, et al. (2009). Increased migration of Langerhans cells in response to HPV16 E6 and E7 oncogene silencing: role of CCL20. Cancer Immunol. Immunother. 58: 39-47.

Da Silva DM, Movius CA, Raff AB, Brand HE, et al. (2014). Suppression of Langerhans cell activation is conserved amongst human papillomavirus alpha and beta genotypes, but not a micro genotype. Virology 452-453: 279-286.

Du K, Gong HY and Gong ZM (2014). Influence of serum VEGF levels on therapeutic outcome and diagnosis/prognostic value in patients with cervical cancer. Asian Pac. J. Cancer Prev. 15: 8793-8796.

Durzynska J (2014). IGF axis and other factors in HPV-related and HPV-unrelated carcinogenesis (review). Oncol. Rep. 32: $2295-2306$

Gan L, Jia R, Zhou L, Gou J, et al. (2014). Fusion of CTLA-4 with HPV16 E7 and E6 enhanced the potency of therapeutic HPV DNA vaccine. PloS One 9: e108892.

Giannini SL, Hubert P, Doyen J, Boniver J, et al. (2002). Influence of the mucosal epithelium microenvironment on Langerhans cells: implications for the development of squamous intraepithelial lesions of the cervix. Int. J. Cancer 97: 654-659.

Guess JC and McCance DJ (2005). Decreased migration of Langerhans precursor-like cells in response to human keratinocytes expressing human papillomavirus type $16 \mathrm{E} 6 / \mathrm{E} 7$ is related to reduced macrophage inflammatory protein-3alpha production. J. Virol. 79: 14852-14862.

He H, Liu X, Wang D, Wang Y, et al. (2014). SAHA inhibits the transcription initiation of HPV18 E6/E7 genes in HeLa cervical cancer cells. Gene 553: 98-104.

Hussain S, Nasare V, Kumari M, Sharma S, et al. (2014). Perception of human papillomavirus infection, cervical cancer and HPV vaccination in North Indian population. PloS One 9: e112861

Nahvijou A, Hadji M, Marnani AB, Tourang F, et al. (2014). A systematic review of economic aspects of cervical cancer screening strategies worldwide: discrepancy between economic analysis and policymaking. Asian Pac. J. Cancer Prev. 15: 8229-8237.

Nakayama Y, Asagoe K, Yamauchi A, Yamamoto T, et al. (2011). Dendritic cell subsets and immunological milieu in inflammatory human papilloma virus-related skin lesions. J. Dermatol. Sci. 63: 173-183. 
Nessa A, Rashid MH, Jahan M, Ferdous NE, et al. (2014). Role of the HPV DNA test in follow-up of treated cervical intraepithelial neoplasia in Bangladesh. Asian Pac. J. Cancer Prev. 15: 8063-8067.

Niebler M, Qian X, Höfler D, Kogosov V, et al. (2013). Post-translational control of IL-1beta via the human papillomavirus type 16 E6 oncoprotein: a novel mechanism of innate immune escape mediated by the E3-ubiquitin ligase E6-AP and p53. PLoS Pathog. 9: e1003536.

Rios-Yuil JM, Herrera-Gonzalez NE, Aguilar-Faisal JL, Lara-Padilla E, et al. (2014). The molecular fingerprint of human papillomavirus infection and its effect on the Langerhans cell population in squamous cell carcinomas of the genital skin. Indian J. Dermatol. Venereol. Leprol. 80: 381.

Skaaby T, Husemoen LL, Jørgensen T, Johansen JD, et al. (2014). Associations of filaggrin gene loss-of-function variants and human papillomavirus-related cancer and pre-cancer in Danish adults. PloS One 9: e99437.

Sperling T, Oldak M, Walch-Rückheim B, Wickenhauser C, et al. (2012). Human papillomavirus type 8 interferes with a novel C/EBPbeta-mediated mechanism of keratinocyte CCL20 chemokine expression and Langerhans cell migration. PLoS Pathog. 8: e1002833.

Wang X, Gao XH, Hong Y, Li X, et al. (2010). Local hyperthermia decreases the expression of CCL-20 in condyloma acuminatum. Virol. J. 7: 301.

Xu LD, Muller S, Thoppe SR, Heliborg F, et al. (2014). Expression of the p53 target Wig-1 is associated with HPV status and patient survival in cervical carcinoma. PloS One 9: e111125.

Zamaniah WW, Mastura M, Phua C, Adlinda A, et al. (2014). Definitive concurrent chemoradiotherapy in cervical cancer - a University of Malaya Medical Centre experience. Asian Pac. J. Cancer Prev. 15: 8987-8992.

Zeng C, Chen L, Yang Z and Sun S (2014). The close correlation between heparanase and COX-2 expression in lymphangiogenesis of cervical cancer. Med. Oncol. 31: 314. 